\title{
Application of Discrete Fourier Series for Surrogate Modeling
}

\author{
Eldin Wee Chuan Lim, Senior Member, IACSIT
}

\begin{abstract}
A new methodology for deriving surrogate models for solving black-box optimization problems has been developed in this study. A discrete Fourier series was derived from the conventional Fourier series formulation and principles associated with the discrete Fourier transform, and used for the reformulation of black-box objective functions as continuous functions. A stochastic global optimization technique known as Particle Swarm Optimization (PSO) was then applied to locate the global optimal solutions of the continuous functions derived. The methodology was applied to the solution of a black-box optimization problem that was simulated on the basis of the Himmelblau function.
\end{abstract}

Index Terms-Black-box optimization, discrete Fourier series, global optimization, particle swarm optimization, surrogate models

\section{INTRODUCTION}

Black-box optimization refers to the maximization or minimization of an objective function over a set of feasible parameter values where the objective function cannot be evaluated analytically. Such optimization problems may arise in situations where a complex system whose behavior is not well understood and not formally described needs to be optimized [1]. In such problems, values of the objective function may have to be measured, estimated or evaluated from simulations and the domain(s) of the objective function and/or feasible region is usually non-continuous in nature. Such optimization problems are ubiquitous in engineering, operations research and computer science. However, research on black-box optimization techniques seems to be limited largely to within the domain of theoretical computer science [2]. Here, a new approach for solving black-box optimization problems that draws analogy from concepts rooted in the realm of discrete optimization is presented.

As is typical in such problems, the objective function is composed of a set of points which may be assumed to be generated from simulations or experiments. It may be assumed that an analytical function representation of the objective function is unavailable, regardless of whether such a function is derivable by other theoretical or modeling means. In principle, the full objective function may be derived empirically by conducting an infinite number of such simulations or experiments to obtain the complete set of data

Manuscript received October 25, 2012; revised November 27, 2012. This work was supported in part by the Bill \& Melinda Gates Foundation through the Grand Challenges Explorations Initiative under Grant R-279-000-346-720.

E. W. C. Lim is with the Department of Chemical \& Biomolecular Engineering, National University of Singapore (e-mail: chelwce@nus.edu.sg). points. However, such an approach is almost always impractical as these simulations or experiments are usually expensive to conduct. The goal of the optimization process is to obtain the value(s) of the independent variable(s) which will maximize (or minimize) the objective function, keeping in mind that the optimal solution may not correspond to any of the available data points representing the objective function. Currently available methods that make use of linearization, smoothing or averaging techniques to derive an equivalent continuous formulation of the black-box objective function tend to distort the objective function and are approximations to the true analytical model at best. On the other hand, it is also well-established that a function (continuous or otherwise) may be represented as a Fourier series consisting of a large number of trigonometric terms. In the limit that the number of such terms approaches infinity, the Fourier series will converge exactly to the original function it represents, thus making it effectively a universal mathematical regression technique. Based on this premise, it was conjectured that a Fourier series representation of a black-box objective function may be derived that will correspond exactly or almost exactly to the true analytical model and which also effectively converts the optimization problem involving black-box functions into a continuous problem. A stochastic global optimization method may then be applied to solve the continuous optimization problem. Such an approach exploits the superior regression capabilities of the Fourier series method towards solving black-box optimization problems and has recently been applied successfully towards solving a long-standing problem in the area of chemical defense referred to as the chemical plume tracing problem $[3,4]$. In the next section, the formulation of the Fourier series method as applied to black-box functions will be derived and the basic principles of a stochastic method, Particle Swarm Optimization, which will be applied towards solving the resulting continuous optimization problem will be introduced.

\section{COMPUtATIONAL MethoD}

\section{A. Discrete Fourier Analysis}

A periodic function $\mathrm{f}(x)$ with period $\mathrm{L}$ can be expressed as a Fourier series given by the expression:

$$
f(x)=a_{o}+\sum_{n=1}^{\infty}\left[a_{n} \cos \left(\frac{n \pi x}{L}\right)+b_{n} \sin \left(\frac{n \pi x}{L}\right)\right]
$$

where $n=1,2,3, \ldots$ and the coefficients are given by the integrals: 


$$
\begin{gathered}
a_{o}=\frac{1}{L} \int_{0}^{L} f(x) d x \\
a_{n}=\frac{2}{L} \int_{0}^{L} f(x) \cos \left(\frac{n \pi x}{L}\right) d x \\
b_{n}=\frac{2}{L} \int_{0}^{L} f(x) \sin \left(\frac{n \pi x}{L}\right) d x
\end{gathered}
$$

For mathematical analysis, the Fourier series may also be expressed in exponential form with complex coefficients:

$$
f(x)=\sum_{n=-\infty}^{\infty} \alpha_{n} e^{i\left(\frac{2 \pi n x}{L}\right)}
$$

where $\alpha_{n}=\frac{1}{2}\left(a_{n}-i b_{n}\right), n=0, \pm 1, \pm 2, \ldots$ and $\alpha_{n}$ is given by:

$$
\alpha_{n}=\frac{1}{L} \int_{-L / 2}^{L / 2} f(x) e^{-i\left(\frac{2 \pi n x}{L}\right)} d x
$$

By the convolution theorem, it may be shown that the coefficients $\alpha_{n}$ of the Fourier series expansion of $\mathrm{f}(x)$ are equal to the values of the corresponding Fourier transform of $\mathrm{f}(x)$, denoted as $\mathrm{F}(f)$, evaluated at $n / L$. In other words, the Fourier transform of a periodic function is then an infinite set of sinusoids (i.e., an infinite sequence of equidistant impulses) with amplitudes $\mathrm{F}(n / L)$ [5].

Consider a continuous function $\mathrm{f}(x)$ and its Fourier transform $\mathrm{F}(f)$ which are discretized by sampling and truncation so that only a finite number of points, say $N$, are considered. If it is assumed that the $N$ samples of the original function $\mathrm{f}(x)$ are one period of a periodic waveform, the Fourier transform of this periodic function is given by the $N$ samples as computed by the expression:

$$
F\left(\frac{n}{N \ell}\right)=\sum_{k=0}^{N-1} f(k \ell) e^{-i\left(\frac{2 \pi n k}{N}\right)}
$$

where $\ell=L / N$ and $n=0,1, \ldots, N-1$. This is the well-established discrete Fourier transform [5].

The sampling theorem states that if the Fourier transform of a function $\mathrm{f}(x)$ is zero for all frequencies greater than a certain frequency $f_{c}$, then the continuous function $\mathrm{f}(x)$ can be uniquely determined from knowledge of its sampled values. Consider a discrete function containing $N$ data points for which the corresponding continuous function is to be determined. Without loss of generality, the smallest interval between any two adjacent data points may be used to define the highest frequency component of the Fourier transform of the corresponding continuous function. Further, the domain of the discrete function, say $0 \leq x \leq \mathrm{L}$, may be assumed to be one period of a periodic waveform of the corresponding continuous function. This allows Eq. (7) to be applied for calculating values of the discrete Fourier transform of the discrete function and by the convolution theorem mentioned earlier, these will be equal to the coefficients of the Fourier series expansion of the corresponding continuous function. By this argument, the following expression for the double Fourier sine series of a two-dimensional continuous function may be derived from its discrete counterpart:

$$
\begin{gathered}
f(x, y)=\sum_{m=1}^{M} \sum_{n=1}^{N} a_{m n} \sin \left(\frac{m \pi x}{L_{x}}\right) \sin \left(\frac{n \pi y}{L_{y}}\right) \\
a_{m n}=\frac{4}{M N} \sum_{h=1}^{M} \sum_{k=1}^{N} f\left(\frac{h}{M}, \frac{k}{N}\right) \sin \left(\frac{m \pi h}{M}\right) \sin \left(\frac{n \pi k}{N}\right)
\end{gathered}
$$

\section{B. Particle Swarm Optimization}

The most challenging global optimization problems are those that do not contain any known structure that can be exploited. However, stochastic methods which generally require few assumptions on the optimization problem are particularly suited for such problems. The Particle Swarm Optimization (PSO) algorithm is a population based search algorithm based on the simulation of the social behavior of birds within a flock [6]. The initial intent of the particle swarm concept was to graphically simulate the graceful and unpredictable choreography of a bird flock [7], with the aim of discovering patterns that govern the ability of birds to fly synchronously and to suddenly change direction with a regrouping in an optimal formation. In PSO, a swarm of individuals are referred to as particles, with each representing a potential solution. Each particle is flown through a hyperdimensional search space in such a manner that the behavior of each particle is influenced by its own experience as well as those of its neighbors. The concept behind PSO is based on the social-psychological tendency of individuals to emulate the success of other individuals and therefore represents a kind of symbiotic cooperative algorithm. The position and velocity of each particle, $\mathrm{P}_{\mathrm{i}}$, in the swarm are updated at each time step according to the following equations:

$$
\begin{gathered}
X_{i}(t)=x_{i}(t-1)+v_{i}(t) \Delta t \\
v_{\mathrm{i}}(t)=\varphi v_{i}(t-1)+\rho_{1}\left\{x_{p b e s t, i}-x_{i}(t-1)\right\}+\rho_{2}\left\{x_{\text {gbest }}-x_{i}(t-1)\right\}
\end{gathered}
$$

where $x_{i}(t)$ and $v_{i}(t)$ are the position and velocity of particle $P_{i}$ at time $\mathrm{t}$ respectively, $\Delta \mathrm{t}$ is the time step which is assigned an arbitrary value of $1.0, \phi$ is an inertia weight, $\rho_{1}$ and $\rho_{2}$ are random variables, $x_{p b e s t, i}$ is the position giving the best performance of $P_{i}$ up to time $\mathrm{t}$ and $x_{\text {gbest }}$ is the position giving the globally best performance of the entire swarm up to the current time. In the current implementation, particles that attempt to travel out of the domain of the function to be optimized are constrained to remain stationary at the edge of the domain until they reverse their direction of motion.

The second and last terms above are commonly referred to as the cognitive and social components respectively. The further away a particle is from its own best solution and the global best solution, the larger the driving force provided by these components to move the particle towards the best solutions. The random variables $\rho_{1}$ and $\rho_{2}$ are defined as $\rho_{1}=$ 
$r_{1} c_{1}$ and $\rho_{2}=r_{2} c_{2}$, with $r_{1}$ and $r_{2}$ randomly selected from a uniform distribution $U(0,1)$ and $c_{1}$ and $c_{2}$ are positive acceleration constants. Kennedy [8] has shown that $c_{1}+c_{2} \leq$ 4 is a necessary condition in this formulation. Velocities and positions tend to diverge to infinity otherwise. The inertia weight $\phi$ provides improved performance of the algorithm by controlling the influence of previous velocities on the new velocity. Physically, larger inertia weights cause larger exploration of the search space and vice versa. However, studies have also shown that the PSO algorithm does not converge for all combinations of the inertia weight and the acceleration constants $c_{1}$ and $c_{2}$. To ensure convergence, the following relation must hold:

$$
\varphi>\frac{1}{2}\left(c_{1}+c_{2}\right)-1
$$

with $\phi \leq 1$. The PSO algorithm tends to exhibit cyclic or divergent behavior if the above relation is not satisfied. In the present study, values of the above parameters used were $c_{1}=$ $1.0, c_{2}=1.0$ and $\phi=0.5$.

\section{RESUlTS AND DISCUSSION}

The above proposed methodology for solving black-box optimization problems was applied to a well-known optimization problem to validate the applicability of the proposed approach of combining discrete Fourier analysis with Particle Swarm Optimization for solving black-box optimization problems. The two-dimensional Himmelblau function was used as a test function to simulate a black-box optimization problem:

$$
\mathrm{f}(x, y)=\left(x^{2}+y-11\right)^{2}+\left(x+y^{2}-7\right)^{2}
$$

For simulating a black-box optimization problem, it was assumed that the above function was unknown analytically and only function values at specific points within the domains were available. The two-dimensional continuous equivalent of this black-box function was then derived by applying the discrete double Fourier sine series. Subsequently, the global minimum point located by applying the PSO algorithm to this discrete double Fourier sine series representation of the Himmelblau function was then compared with the true global minimum of the function.

Fig. 1a shows the two-dimensional Himmelblau function in the domains $0 \leq x \leq 4$ and $0 \leq y \leq 4$ where the global minimum point was known to exist at the position $(x, y)=(3$, 2 ). Values of the function at about 100 points indicated by the white dots were assumed to be known while the rest of the function was assumed to be unavailable. This set of discrete data points was then treated as part of a black-box function whose global minimum point was to be located. The discrete double Fourier sine series was first applied to this set of discrete data points and Figure $1 \mathrm{~b}$ shows that the discrete Fourier series representation obtained was qualitatively similar to the original Himmelblau function although quantitative differences may be discerned. The PSO algorithm with 100 particles was then applied to this discrete Fourier series representation of the original function and Fig.
2 shows that the particles were able to converge gradually over about 300 iterations. The position of the global minimal point reported at the end of 300 iterations was $(x, y)=(3.00$, 2.14) which was in close agreement with the actual global minimum of the Himmelblau function. This illustrates the applicability of the methodology for solving black-box optimization problems.
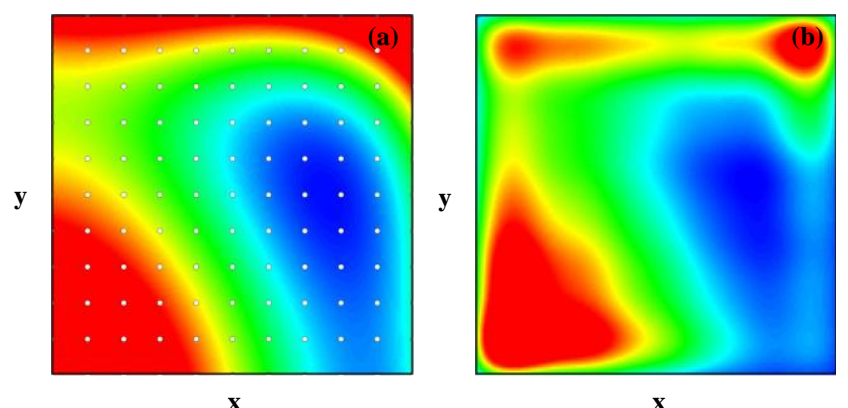

Fig. 1. (a) The Himmelblau function in the domains $0 \leq x \leq 4$ and $0 \leq y \leq 4$. The color contour scales linearly from blue (0) to red (100). The function was assumed to be unknown analytically and white dots indicate positions at which function values were available for black-box optimization. (b) Continuous equivalent of this black-box function derived by applying a discrete double Fourier sine series to this set of about 100 discrete data points.
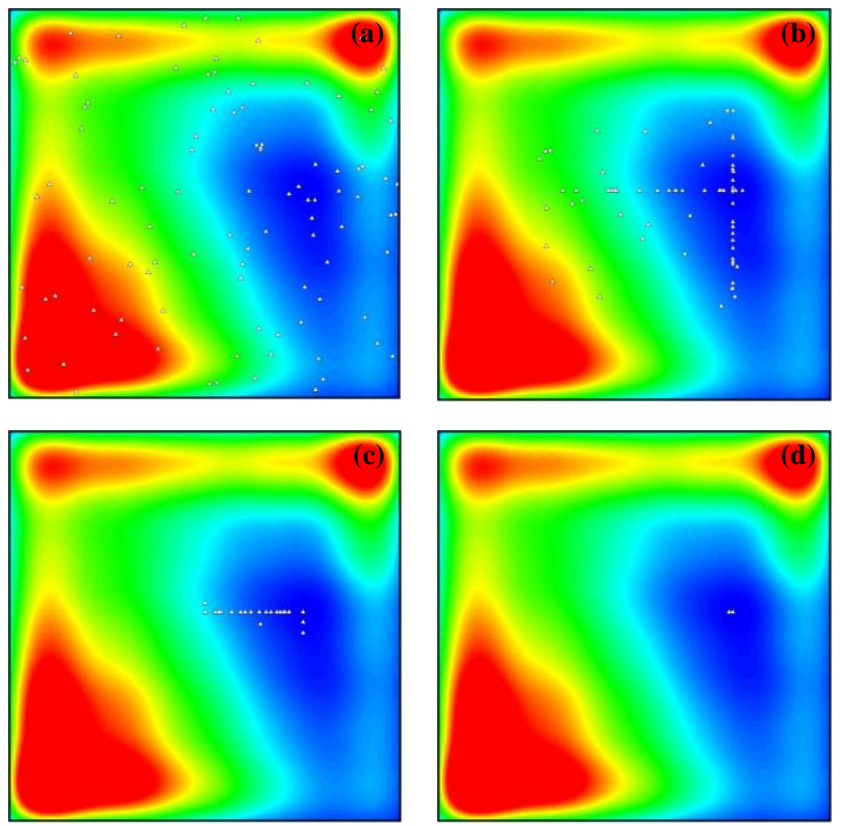

Fig. 2. Global optimization by the PSO algorithm on the discrete double Fourier sine series representation of the Himmelblau function generated from about 100 discrete data points. (a) 100 PSO particles were initially randomly distributed over the domain. Snapshots of the optimization process show positions of the particles after (b) 100 , (c) 200 and (d) 300 iterations of the PSO algorithm.

\section{CONCLUSIONS}

A new methodology for solving black-box optimization problems by the continuous approach has been developed in this study. A discrete Fourier series method was derived from the conventional Fourier series formulation and principles associated with the discrete Fourier transform and used for re-formulation of black-box objective functions as continuous functions. A stochastic global optimization technique known as Particle Swarm Optimization (PSO) was then applied to locate the global optimal solutions of the 
continuous functions derived. The methodology developed here was applied towards solving a black-box optimization problem that was simulated based on the Himmelblau function. The discrete Fourier series method coupled to the PSO algorithm is a promising methodology for solving black-box optimization problems via the continuous approach.

\section{REFERENCES}

[1] S. Droste, T. Jansen, and I. Wegener, "Optimization with randomized search heuristics - the (A) NFL theorem, realistic scenarios, and difficult functions," Theoretical Computer Science, vol. 287, pp. $131-144,2002$.

[2] S. Droste, T. Jansen, and I. Wegener, "Upper and lower bounds for randomized search heuristics in black-box optimization," Theory of Computing Systems, vol. 39, pp. 525-544, 2006.

[3] E. W. C. Lim, "Application of particle swarm optimization to Fourier series regression of non-periodic data," Industrial \& Engineering Chemistry Research, vol. 50, pp. 2307-2322, 2011.

[4] E. W. C. Lim and J. R. New, "Black-box optimization by Fourier analysis and swarm intelligence," Journal of Chemical Engineering of Japan, vol. 45, pp. 417-428, 2012.

[5] E. O. Brigham, The Fast Fourier Transform, Prentice-Hall, Inc., Englewood cliffs, N. J, 1974.

[6] A. P. Engelbrecht, Computational Intelligence an Introduction, England: John Wiley and Sons Ltd, 2002.
[7] J. Kennedy and R. C. Eberhart, "Particle swarm optimization," in Proceedings of the IEEE International Conference on Neural Networks, vol. 4, pp. 1942-1948, 1995

[8] J. Kennedy, "The behavior of particles," in Proceedings of the 7th International Conference on Evolutionary Programming, in: V.W. Porto, N. Saravanan, and D. Waagen (Eds.), pp. 581-589, 1998.

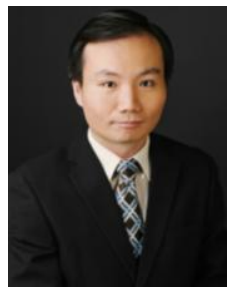

Eldin Wee Chuan Lim received his B.Eng. (Hons), M.Eng. and Ph.D. degrees, all in chemical engineering, from the National University of Singapore. He is a Lecturer in the Department of Chemical \& Biomolecular Engineering at the National University of Singapore. Dr. Lim received a Young Researcher Award presented by the European Regional Section of the International Association for Statistical Computing (ERS-IASC), the Woodside Energy Award and the Hutchinson Award at the 2010 SECAU Security Congress and the Frontier Award presented by the Fluid \& Particle Processing Division of The Society of Chemical Engineers, Japan. He has served as a Book Editor for InTech Open Access Publisher and as a Member of the Editorial Boards for the Elsevier journal, Advanced Powder Technology, the American Journal of Fluid Dynamics published by Scientific \& Academic Publishing, the Journal of Chemical Engineering \& Process Technology published by OMICS Publishing Group, and the Journal of Chemistry and Chemical Engineering published by David Publishing Company. He is currently the Editor-in-Chief for the International Journal of Chemical Engineering and Applications published by IACSIT Press. 